\title{
Besoins nutritionnels des bactéries lactiques : interactions entre flux de carbone et d'azote
}

\author{
P Loubière, L Novak, M Cocaign-Bousquet, ND Lindley
}

Centre de bio-ingénierie Gilbert-Durand (URA CNRS 544 \& Lab Assoc Inra), Institut national des sciences appliquées, complexe scientifique de Rangueil, 31077 Toulouse cedex, France

\begin{abstract}
Résumé - La détermination des exigences nutritionnelles de Lactococcus lactis subsp lactis montre que la souche IL 1403 isolée d'un milieu laitier est auxotrophe pour neuf acides aminés et cinq vitamines. En revanche, la souche NCDO 2118 isolée d'un milieu végétal est, d'après la technique d'omission individuelle, prototrophe vis-à-vis des acides aminés, mais exige pourtant six acides aminés et cinq vitamines pour sa croissance. À partir du milieu minimum contenant ces six acides aminés, l'enlèvement du glutamate provoque un temps de latence de 2 jours et un ralentissement de croissance. La difficulté à synthétiser le glutamate à partir du métabolisme central est due à la fois à la portion de cycle de Krebs entre l'oxaloacétate et l' $\alpha$-cétoglutarate, et à la glutamate déshydrogénase. Vis-à-vis des acides aminés branchés, outre la confirmation de la régulation de l'acétolactate décarboxylase par la leucine, il est mis en évidence une régulation de(s) acétohydroxyacide synthase(s) par la thréonine et la thiamine. Le métabolisme de la sérine est régulé, tant au niveau de sa biosynthèse que de sa consommation, par d'autres acides aminés ou vitamines. En effet, sa consommation est douze fois plus importante dans le milieu minimum contenant six acides aminés que dans un milieu en contenant dix-huit. Son devenir, déterminé par incubation en présence de ${ }^{14} \mathrm{C}$-sérine, montre qu'elle est désaminée puis transformée en lactate.
\end{abstract}

\section{Lactococcus lactis / acide aminé / croissance / métabolisme / régulation}

Summary - Nutritional requirements of lactic acid bacteria: interactions between carbon and nitrogen flux. Nutritional requirements have been determined for two strains of Lactococcus lactis subsp lactis. The IL 1403 dairy strain, was shown to be auxotroph for nine amino acids and five vitamins, while the NCDO 2118 strain isolated from vegetable matter was prototroph for all amino acids when using the single omission technique, but nevertheless required six amino acids and five vitamins for growth in minimal medium. In such a medium, the suppression of glutamate led to a lag phase of about 2 days and a decrease of the growth rate. The problem of glutamate synthesis is linked to the part of Krebs cycle between oxaloacetate and $\alpha$-ketoglutarate, and to the glutamate dehydrogenase. Concerning the branched-chain amino acids, not only has the regulation of acetolactate decarboxylase been confirmed, but the regulation of acetohydroxy acid synthase(s) by threonine and thiamine has also been observed. Both the consumption and the synthesis of serine are regulated by other amino acids or vitamins. Serine consumption is about 12 times higher in the minimal medium than in a medium containing 18 aminoacids. The study of ${ }^{14} \mathrm{C}$-serine distribution showed that it is deaminated and transformed to lactate.

Lactococcus lactis / amino acid / growth / metabolism / regulation 


\section{INTRODUCTION}

Malgré une importance considérable dans le domaine agro-alimentaire, la croissance des bactéries lactiques reste un phénomène fastidieux qui nécessite un apport complexe, aussi bien qualitativement que quantitativement, en éléments nutritifs. Outre les besoins en oligo-éléments, vitamines et nucléotides, leurs exigences nutritionnelles les plus remarquables concernent certainement les besoins en acides aminés. II est, en effet, clairement établi qu'aucune d'entre elles n'est capable de se développer aux dépens d'azote inorganique. Le problème est souvent résolu par l'utilisation de milieux de culture complexes à base de peptone, extrait de levure et autre tryptone. Cependant, de tels milieux ne permettent ni d'identifier les véritables exigences nutritionnelles, ni de caractériser les voies métaboliques empruntées. Si l'étude des peptidases et protéases des bactéries lactiques, particulièrement mises à contribution lors de la croissance en milieux complexes ou naturels (lait), est bien avancée, le rôle des acides aminés en relation avec la biosynthèse cellulaire est complètement négligé.

Relativement peu d'études ont été consacrées à la détermination complète des exigences nutritionnelles des bactéries lactiques, en particulier en acides aminés. Elles sont fondées sur la technique d'omission individuelle de chacun des éléments du milieu. Cette technique a été utilisée par Reiter et Oram (1962) pour déterminer les auxotrophies de plusieurs souches de Lactococcus lactis. Ils montrent que toutes les souches testées possèdent au moins des auxotrophies pour le glutamate, la valine, la leucine, l'isoleucine, la méthionine et l'histidine. Cette information est cependant insuffisante pour formuler un milieu de croissance simplifié, comme l'ont montré Jensen et Hammer (1993). D'autres acides aminés que les six identifiés par Reiter et Oram ont dû être rajoutés au milieu pour obtenir une croissance de $L$ lactis subsp lactis.

La mise en évidence d'une auxotrophie par la technique d'omission individuelle indique une déficience de la voie métabolique impliquée. Morishita et al $(1974,1981)$ ont montré que, chez Lactobacillus casei, ces déficiences sont, en général, récupérables par mutagenèse, sauf dans le cas du glutamate. II semble que l'impossibilité de synthétiser le glutamate soit un caractère général des bactéries lactiques, jusqu'à présent systématiquement observé.

L'étude la plus récente et la plus complète porte sur la détermination de la totalité des exigences nutritionnelles (oligo-éléments, vitamines, nucléotides et acides aminés) de deux souches de $L$ lactis subsp lactis, NCDO 2118 et IL1403, et la formulation d'un milieu minimal pour la croissance de chaque souche (Cocaign-Bousquet et al, 1995). Ces résultats sont complétés et discutés dans cette étude, et des données originales sur le métabolisme des acides aminés chez les bactéries lactiques sont présentées.

\section{MATÉRIELS ET MÉTHODES}

Les souches utilisées dans cette étude sont Lactococcus lactis subsp lactis NCDO 2118 et IL 1403 , isolées de milieu végétal et laitier respectivement. Elles sont conservées à $-80^{\circ} \mathrm{C}$ dans du milieu chimiquement défini (MCD) décrit par Otto et al (1983) et modifié par Poolman et Konings (1988), supplémenté de glycérol $(20 \%)$. Ce milieu MCD sert également de milieu de référence pour la croissance. Un milieu synthétique simplifié (MS10) mis au point par Cocaign-Bousquet et al (1995) est également utilisé comme référence (tableau I). Les cultures sont réalisées à $30^{\circ} \mathrm{C}, \mathrm{pH} 6,6$, sous atmosphère d'azote, et agitées à $250 \mathrm{rpm}$. Les conditions de culture et les méthodes analytiques sont identiques à celles précédemment décrites (Cocaign-Bousquet et al, 1995).

Les acides aminés des surnageants de fermentation sont dosés sur un amino-quant Hewlett-Packard 1090, après dérivatisation en pré- 
colonne par l'ortho-phtalique aldéhyde en présence d'acide 3-mercaptopropionique à $\mathrm{pH} 10$. La séparation s'effectue sur une colonne $\mathrm{C} 18$, et la détection par spectrophotométrie à $338 \mathrm{~nm}$. La concentration en ion ammonium est mesurée grâce à une électrode ionsélective Orion reliée à un module Orion 710A pH-mV-ISA-mètre.

\section{RÉSULTATS ET DISCUSSION}

\section{Exigences nutritionnelles et formulation d'un milieu minimum}

La technique d'omission individuelle de chacun des éléments du milieu de culture (MCD) met en évidence un comportement radicalement différent pour les deux souches de $L$ lactis testées. L'omission de chacun des sept acides aminés suivants, glutamate, isoleucine, leucine, valine, méthionine, histidine et arginine, empêche toute croissance de la souche IL 1403. L'omission de chacun des autres acides aminés, si elle provoque quelquefois un ralentissement de la croissance, n'est jamais associée à un temps de latence avant croissance. De même, une auxotrophie pour cinq vitamines, biotine, nicotinate, pantothenate, pyridoxamine et riboflavine, est mise en évidence par cette technique. On observe donc pour cette souche un comportement "classique" où une exigence traduit une impossibilité totale de synthèse de l'élément en question, c'est-à-dire une déficience dans une voie métabolique. II est cependant important de remarquer que la croissance est impossible dans un milieu contenant les cinq vitamines et les sept acides aminés identifiés comme indispensables. Le rajout de sérine et de thréonine s'avère alors obligatoire, constituant ainsi le milieu minimal (MS15) permettant la croissance de la souche IL 1403 (tableau I). Ce résultat illustre le fait que la technique d'omission individuelle en elle-même est insuffisante pour formuler une composition de milieu de culture, comme l'avaient constaté Jensen et Hammer (1993) sur la base des résultats de Reiter et Oram (1962).

La même technique appliquée à la souche NCDO 2118 montre une exigence en biotine, nicotinate et pantothenate. Concernant, les acides aminés, la valine est nécessaire uniquement en présence de leucine (la croissance est possible en absence de leucine et valine). Hormis cet effet (discuté ultérieurement), aucun acide aminé ne s'avère indispensable, confirmant en cela le caractère prototrophe de cette souche. Par contre, un temps de latence est remarqué en absence de glutamate, leucine ou méthionine. Malgré cette apparente prototrophie, la croissance est impossible dans un milieu sans acides aminés et contenant les trois vitamines indispensables. Une étude de la croissance sur des milieux graduellement simplifiés a été nécessaire pour identifier tous les éléments nutritifs requis pour la constitution d'un milieu minimal. Certaines exigences croisées ont ainsi été mises en évidence ; la suppression des bases nucléotidiques entraine une exigence en pyridoxamine, bien qu'elle ne soit pas impliquée directement dans la biosynthèse des nucléotides. Ces résultats ont permis la formulation d'un milieu synthétique simplifié (MS10, tableau I), mais qui contient encore la totalité des acides aminés. Le milieu minimal qui permette une croissance sans phase de latence doit contenir glutamate, isoleucine, leucine, valine, méthionine et sérine, mais aussi de la riboflavine dont la nécessité n'apparaît qu'en présence de cette composition simplifiée en acides aminés. Le milieu minimal ainsi composé est le MS13 (tableau I). Une étude complémentaire de la croissance et de la consommation des acides aminés en fermentation de la souche NCD0 2118 sur milieu MS13 a permis d'optimiser la concentration de chaque acide aminé en fonction de sa consommation réelle, pour une concentration initiale de glucose de $10 \mathrm{~g} / \mathrm{l}$ (milieu 
MS14, tableau I). Ces résultats montrent que la riboflavine est impliquée, directement ou indirectement, dans la synthèse de l'un des 12 acides aminés retirés du milieu MS10. D'autre part, malgré la présence potentielle de toutes les voies de synthèse des acides aminés, leur fonctionnement dépend de la composition du milieu en acides aminés (ou vitamines). II existe donc un ensemble complexe de régulations entre acides aminés de leurs propres voies de biosynthèse, qui montre que la définition de prototrophie/auxotrophie est en partie dépendante de la composition du milieu de culture.

\section{Comportement de L lactis NCDO 2118 vis-à-vis des acides aminés essentiels}

Le comportement particulier de la souche NCDO 2118 vis-à-vis des acides aminés

Tableau I. Composition des milieux synthétiques.

Synthetic media composition.

\begin{tabular}{|c|c|c|c|c|}
\hline Constituant & MS10 & MS15 & MS13 & MS14 \\
\hline Glucose & 10 & 10 & 10 & 10 \\
\hline$\left(\mathrm{NH}_{4}\right)_{2} \mathrm{SO}_{4}$ & 0,18 & - & - & - \\
\hline $\mathrm{KH}_{2} \mathrm{PO}_{4}$ & $9(3)^{1}$ & $9(3)^{1}$ & $9(3)^{1}$ & $9(3)^{1}$ \\
\hline $\mathrm{K}_{2} \mathrm{HPO}_{4}$ & $7,5(2,5)^{1}$ & $7,5(2,5)^{1}$ & $7,5(2,5)^{1}$ & $7,5(2,5)^{1}$ \\
\hline $\mathrm{MgCl}_{2}, 6 \mathrm{H}_{2} \mathrm{O}$ & 0,2 & 0,2 & 0,2 & 0,2 \\
\hline Pyridoxamine & 0,005 & 0,005 & 0,005 & 0,005 \\
\hline Biotine & 0,01 & 0,01 & 0,01 & 0,01 \\
\hline Acide nicotinique & 0,001 & 0,001 & 0,001 & 0,001 \\
\hline Ca-pantothenate & 0,001 & 0,001 & 0,001 & 0,001 \\
\hline Riboflavine & - & 0,001 & 0,001 & 0,001 \\
\hline Alanine & 0,24 & - & - & - \\
\hline Arginine & 0,12 & 0,12 & - & - \\
\hline Asparagine & 0,34 & - & - & - \\
\hline Cystéine & 0,17 & - & - & - \\
\hline Glutamate & - & 0,10 & 0,10 & 0,30 \\
\hline Glutamine & 0,51 & - & - & - \\
\hline Glycine & 0,17 & - & - & - \\
\hline Histidine & 0,11 & 0,11 & - & - \\
\hline Isoleucine & 0,20 & 0,20 & 0,20 & 0,20 \\
\hline Leucine & 0,47 & 0,47 & 0,47 & 0,47 \\
\hline Lysine-HCl & 0,35 & - & - & - \\
\hline Méthionine & 0,12 & 0,12 & 0,12 & 0,24 \\
\hline Phénylalanine & 0,28 & - & - & - \\
\hline Proline & 0,68 & - & - & - \\
\hline Sérine & 0,34 & 0,34 & 0,34 & 1,02 \\
\hline Thréonine & 0,23 & 0,23 & - & - \\
\hline Tryptophane & 0,05 & - & - & - \\
\hline Tyrosine & 0,29 & - & - & - \\
\hline Valine & 0,33 & 0,33 & 0,33 & 0,16 \\
\hline
\end{tabular}

Concentrations exprimées en g/l. ${ }^{1}$ : Concentrations utilisées en fermenteur à pH régulé.

Concentrations expressed in g/l. ${ }^{1}$ : Concentrations used in a bioreactor with pH regulation. 
indispensables pour une croissance sans phase de latence (fig 1) a été étudié sur la base des différents milieux à notre disposition (MCD, MS10, MS14).

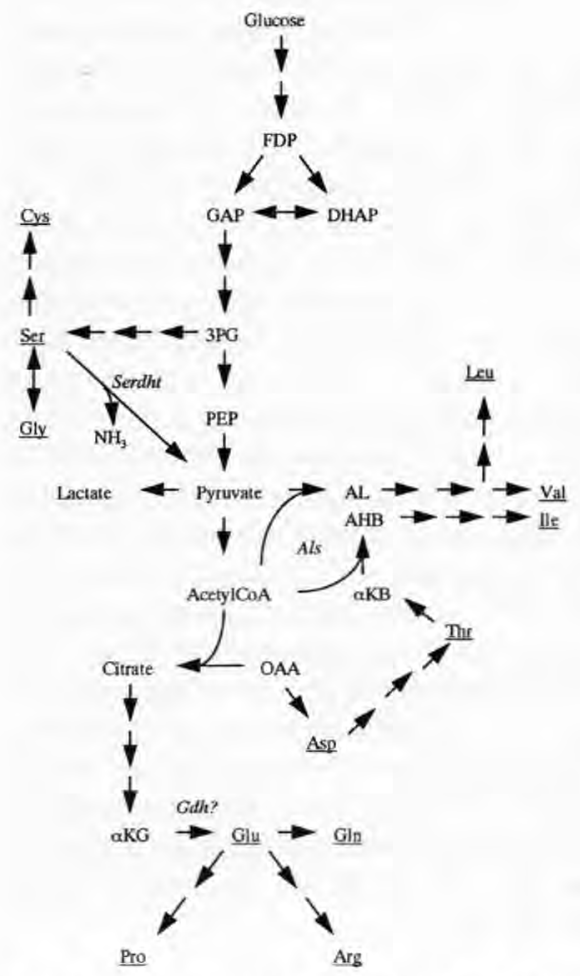

Fig 1. Voies du métabolisme central de Lactococcus lactis représentant les voies de synthèse des acides aminés branchés (isoleucine, leucine, valine) et des familles de la sérine (sérine, glycine, cystéine) et du glutamate (glutamate, glutamine, arginine, proline). Abréviations : FDP : fructose 1,6 diphosphate ; GAP : glycéraldéhyde 3-phosphate; DHAP : dihydroxyacétone phosphate ; $3 P G$ : 3-phosphoglycérate ; PEP : phosphoénolpyruvate ; OAA : oxaloacétate ; $\alpha$ KG : $\alpha$ cétoglutarate ; $\mathrm{AL}$ : acétolactate ; $\mathrm{AHB}$ : acétohydroxybutyrate ; $\alpha$ KB : $\alpha$-cétobutyrate ; Serdht : sérine déshydratase; Als : acétolactate synthase; Gdh: glutamate déshydrogénase. Central metabolic pathways of Lactococcus lactis representing the synthesis pathways for branched-chain amino acids (isoleucine, leucine, valine) and for amino acids of the families of serine (serine, glycine, cysteine) and glutamate (glutamate, glutamine, arginine, proline).

\section{Glutamate}

Parmi les acides aminés de la famille du glutamate (glutamate, glutamine, arginine, proline), aucun problème de biosynthèse n'est observé pour la glutamine, l'arginine et la proline dès lors que le glutamate est présent dans le milieu. En revanche, la suppression du glutamate provoque un temps de latence d'environ $48 \mathrm{~h}$ avant le début de la croissance, et une diminution de moitié du taux de croissance par rapport au milieu témoin. Ces résultats indiquent que le problème de biosynthèse est restreint à la fabrication du glutamate à partir des intermédiaires du métabolisme central. Ils montrent aussi que la biosynthèse du glutamate est possible chez $L$ lactis subsp lactis NCDO 2118 , et donc que toutes les enzymes du cycle de Krebs comprises entre l'oxaloacétate et l' $\alpha$-cétoglutarate, et la glutamate déshydrogénase sont présentes et peuvent être fonctionnelles.

Le temps de latence considérable nécessaire pour la synthèse du glutamate s'est avéré être reproductible et observé aussi bien sur milieu complet (MCD) que sur milieu minimum (MS14). Étant donné le caractère exceptionnel et surprenant de la durée de la phase de latence, une procédure de contrôle a été appliquée; la latence de 2 jours est observée lors du premier transfert d'un milieu MS14 à un milieu MS14 sans glutamate. Comme attendu, le transfert suivant dans ce dernier milieu ne provoque pas de latence. La souche est ensuite transférée deux fois dans le milieu MS14, puis une nouvelle fois dans le milieu MS14 sans glutamate. Une latence de 2 jours est à nouveau observée dans cette condition. Cette procédure confirme d'une part, la reproductibilité de la phase de latence, d'autre part, qu'il n'y a pas eu sélection d'une souche modifiée.

Le ralentissement de croissance $\left(0,12 \mathrm{~h}^{-1}\right.$ au lieu de $0,23 \mathrm{~h}^{-1}$ pour le témoin) et le temps de latence observés en 
l'absence de glutamate restent identiques en présence de citrate ou d'isocitrate dans le milieu. En revanche, un rajout d' $\alpha$-cétoglutarate permet d'obtenir une croissance à un taux de $0,18 \mathrm{~h}^{-1}$ après une latence de seulement $24 \mathrm{~h}$. Un rajout de sulfate d'ammonium dans ce dernier milieu est sans effet sur les paramètres de croissance. La difficulté pour la souche NCDO 2118 à synthétiser le glutamate semble donc provenir à la fois d'une enzyme du cycle de krebs et de la glutamate déshydrogénase, indépendamment pour cette dernière de la disponibilité en azote minéral. L'identification des enzymes concernées et la mesure de leurs activités dans différentes conditions nutritionnelles sont maintenant nécessaires pour préciser les particularités du métabolisme du glutamate dans cette souche originale de lactocoque.

\section{Acides aminés branchés (isoleucine, leucine, valine)}

L'omission de l'isoleucine, la leucine et la valine d'un milieu contenant par ailleurs 15 autres acides aminés, MCD ou MS10, provoque un ralentissement de croissance associé à une phase de latence de 10 et $25 \mathrm{~h}$ respectivement. II semble que l'importance de ce temps de latence soit liée à la composition en vitamines du milieu de culture. La même omission réalisée sur le milieu MS14 empêche toute croissance. Un ou plusieurs acides aminés s'avèrent nécessaires pour permettre la synthèse des acides aminés branchés.

Sur milieu MCD, seul l'enlèvement de la valine bloque la croissance de $L$ lactis NCDO 2118. Cet effet correspond à la régulation de l'acétolactate décarboxylase par la leucine (Godon, 1992 ; Monnet et al, 1994), responsable d'un détournement du flux carboné de l'acétolactate vers l'acétoïne au détriment de la voie de synthèse de valine. L'enlèvement simultané de valine et leucine est sans conséquences sur milieu MCD mais empêche la croissance sur milieu MS14 (tableau II). La biosynthèse de ces deux acides aminés à partir du pyruvate n'est alors possible qu'après rajout de thréonine et de thiamine, certainement nécessaires pour le fonctionnement de l'acétolactate synthase. En effet, cette enzyme est activée par le cétobutyrate directement issu de la thréonine (Eggeling et al, 1987), et fonctionne avec de la thiamine pyrophosphate. D'autre part, la croissance observée sur MS14 dépourvu de leucine, c'est-à-dire dans une condition où l'acétolactate synthase ne doit pas fonctionner, indique que la leucine doit être synthétisée à partir de la valine, après un temps de latence de $36 \mathrm{~h}$. L'enlèvement supplémentaire d'isoleucine en plus de la leucine provoque le même comportement qu'en l'absence de leucine et valine, et doit aussi être expliqué par la nécessité d'une acétohydroxyacide synthase fonctionnelle pour la synthèse de l'isoleucine. Un important travail est aujourd'hui nécessaire pour comprendre les mécanismes de régulation qui gèrent la synthèse des acides aminés branchés, en particulier au niveau de la (ou les) acétohydroxyacide synthase(s).

\section{Sérine}

L'absence de sérine ou de l'ensemble sérine/glycine/cystéine du milieu MCD provoque un ralentissement de la croissance de $L$ lactis NCDO 2118 (avec un taux de croissance résiduel d'environ $40 \%$ ) sans temps de latence. La synthèse de ces trois acides aminés est donc possible dans ces conditions nutritionnelles. En revanche, la croissance est impossible après omission de la sérine du milieu MS14, mais rétablie, bien qu'à une vitesse réduite de moitié, après son remplacement par la glycine. Ces résultats montrent que les étapes du métabolisme entre glycine, sérine et cystéine, 
Tableau II. Effet des acides aminés branchés sur la croissance de Lactococcus lactis subsp lactis NCDO 2118 dans différents milieux de culture.

Effect of branched-chain amino acids on the growth of Lactococcus lactis subsp lactis NCDO 2118 in different media.

\begin{tabular}{|c|c|c|c|c|c|}
\hline Milieu & $-V a l$ & - Leu & $\begin{array}{l}\text { - Leu } \\
\text {-Val }\end{array}$ & $\begin{array}{c}\text {-lle } \\
\text {-Leu }\end{array}$ & $\begin{array}{l}\text {-lle } \\
\text { - Leu } \\
\text {-Val }\end{array}$ \\
\hline $\operatorname{MCD}\left(1 h^{-1}\right)$ & - & + & + & + & + \\
\hline $\operatorname{MS} 14\left(0,23 h^{-1}\right)$ & - & + & - & - & - \\
\hline MS14 + Thr & ND & + & - & - & - \\
\hline MS14 + Thr + thiamine & ND & ND & + & + & + \\
\hline MS14 + thiamine & ND & ND & + & ND & + \\
\hline
\end{tabular}

Abréviations: Ile : isoleucine ; Leu : leucine ; Thr : thréonine ; Val : valine. + : croissance ; - : pas de croissance ; ND : non déterminé.

+: growth; -: no growth; ND: not determined.

sont opérationnelles sur milieu MS14. En revanche, la biosynthèse de sérine à partir du métabolisme central (3P-glycérate) semble exiger la présence d'un acide aminé ou d'une vitamine présents dans le milieu MCD. Ce composé reste à identifier, de même que l'enzyme sur laquelle il agit.

\section{Croissance et métabolisme de $\mathrm{L}$ lactis NCDO 2118 sur milieu minimum}

La consommation du glucose par la souche $L$ lactis NCDO 2118 cultivée sur milieu MS14 répond à la stoechiométrie suivante (en $\mathrm{mmol} / \mathrm{l}$ ) :

100 glucose $+24,5$ ac aminés

8 biomasse +174 lactate +17 formiate + 13 acétate

Le bilan carboné est équilibré à $84 \%$, indiquant que d'autres produits, dont certains apparents en HPLC mais non identifiés, sont formés au cours de la fermentation. Le lactate est le produit majoritaire, caractéristique d'un métabolisme homofermentaire. Le formiate et l'acétate sont formés en quantité significative, tandis qu'aucune production d'éthanol n'est observée.

Alors que chacun des acides aminés du milieu est en partie consommé, la sérine, présente en grande quantité dans le milieu MS14 (19 $\mathrm{mmol} / \mathrm{l}$ ), est épuisée en fin de fermentation. Sa consommation est associée à une importante production de $\mathrm{NH}_{3}$ $(14 \mathrm{mmol} / \mathrm{l})$. II en résulte que le bilan carboné entre les acides aminés consommés et la biomasse produite est largement déficitaire (32\%). En revanche, le bilan azoté entre, d'une part les acides aminés consommés et d'autre part la biomasse et l'ammoniaque produits est équilibré, indiquant qu'aucun sous-produit azoté n'est formé au cours de la fermentation.

La consommation des acides aminés par $L$ lactis NCDO 2118 est comparée en fer- 
mentation sur les milieux MS14 et MS10. La quantité consommée sur MS10 équivaut à la quantité de biomasse formée (bilan de $105 \%$ ). Chacun des acides aminés restant dans le milieu MS14 est deux à trois fois plus consommé que dans le milieu MS10 où 18 acides aminés sont présents, hormis la sérine qui est environ 12 fois plus consommée. II semble qu'il existe une régulation de la consommation de la sérine par un autre acide aminé. Ce phénomène reste pour l'instant inexpliqué.

Une fermentation en milieu MS14 limité en sérine aboutit à un épuisement de cet acide aminé en cours de culture. Comme précédemment, sa consommation est associée à une production de $\mathrm{NH}_{3}$, et d'acétate et formiate comme produits mineurs de fermentation. Après son épuisement, on assiste à une poursuite de la croissance avec, d'une part une transition de la production d'acétate vers celle d'éthanol, d'autre part une reconsommation du $\mathrm{NH}_{3}$ produit. II s'agit ici du seul cas observé de consommation d'azote minéral par cette souche de $L$ lactis. II reste à préciser pourquoi la croissance se poursuit en absence de sérine aux dépens de $\mathrm{NH}_{3}$. Le devenir de la sérine a été plus précisément caractérisé en cultivant la souche dans un milieu MS14 contenant de la ${ }^{14} \mathrm{C}$-sérine. Après incubation, en plus du marquage de la biomasse, du lactate est aussi marqué au ${ }^{14} \mathrm{C}$, indiquant qu'il est un produit de dégradation de la sérine. II semblerait que la sérine soit donc désaminée en pyruvate par la sérine déshydratase, elle-même régulée par un acide aminé, le pyruvate étant alors réduit en lactate par la lactate déshydrogénase. La sérine serait alors un accepteur d'électrons supplémentaire, expliquant l'absence de production d'éthanol pendant sa phase de consommation. Cette hypothèse est actuellement en cours de validation par des expériences supplémentaires.

\section{RÉFÉRENCES}

Cocaign-Bousquet M, Garrigues C, Novak L, Lindley ND, Loubière P (1995) Rational development of a simple synthetic medium for the sustained growth of Lactococcus lactis. J App/ Bacteriol 79, 108-116

Eggeling I, Cordes C, Eggeling L, Sahm H (1987) Regulation of acetohydroxy acid synthase in Corynebacterium glutamicum during fermentation of $\alpha$-ketobutyrate to L-isoleucine. App/ Microbiol Biotechnol 25, 346-351

Godon JJ (1992) Régulation génétique de la synthèse des acides aminés branchés chez Lactococcus lactis. Thèse de l'université Paris XI

Jensen PR, Hammer K (1993) Minimal requirements for exponential growth of Lactococcus lactis. Appl Environ Microbiol 59, 4363-4366

Monnet C, Phalip V, Schmitt P. Divies C (1994) Comparison of $\alpha$-acetolactate synthase and $\alpha$-acetolactate decarboxylase in Lactococcus spp and Leuconostoc spp. Biotechnol Lett 16, 257-262

Morishita T, Fukada T, Shirota M, Yura T (1974) Genetic basis of nutritional requirements in Lactobacillus casei. J Bacteriol 120, 1078-1084

Morishita T, Degushi Y, Yajima M,Sakurai T, Yura T (1981) Multiple nutritional requirements of Lactobacilli: Genetic lesions affecting amino acid biosynthetic pathways. J Bacteriol 148, 64-71

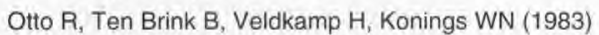
The relation between growth rate and electrochemical proton gradient on Streptococcus cremoris. FEMS Microbiol Lett 16, 69-74

Poolman B, Konings WN (1988) Relation of growth of Streptococcus cremoris to amino acid transport. $\checkmark$ Bacteriol 170, 700-707

Reiter B, Oram JD (1962) Nutritional studies on cheese starters. I. Vitamins and amino acid requirements of single strain starters. J Dairy Res 29, 63-77 